respect to ED utilization, mirrors real-world practice, and allows us to draw conclusions about physician willingness to prevent ARDS.

To conclude, the ED represents an unstudied location and unstudied patient population for the mitigation of ARDS; we propose this is the most logical place to start.

\section{Acknowledgements Conflicts of interest}

There are no conflicts of interest.

\section{References}

1 Rubenfeld GD, Herridge MS. Epidemiology and outcomes of acute lung injury. Chest 2007; 131:554-562.

2 Phua J, Stewart TE, Ferguson ND. Acute respiratory distress syndrome 40 years later: time to revisit its definition. Crit Care Med 2008; 36:2912.

3 Fuller BM, Mohr NM, Drewry AM, Carpenter CR. Lower tidal volume at initiation of mechanical ventilation may reduce progression to acute respiratory distress syndrome - a systematic review. Crit Care 2013; 17:R11.

4 Jones AE, Kline JA. Use of goal-directed therapy for severe sepsis and septic shock in academic emergency departments. Crit Care Med 2005; 33:1888-1889.

5 Needham DM, Bronskill SE, Calinawan JR, Sibbald WJ, Pronovost PJ, Laupacis A. Projected incidence of mechanical ventilation in Ontario to 2026: preparing for the aging baby boomers. Crit Care Med 2005; 33:574-579.

\section{OPEN Retinal imaging: a first report of the retinal microvasculature in acute mild traumatic brain injury}

\begin{abstract}
Charmaine Childs ${ }^{a, c}$, Yi Ting Ong ${ }^{b, d}$, Mya Myint Zuc, Phyo Wai Aung ${ }^{c}$, Carol Y. Cheung ${ }^{b, d}$ and Win Sen Kuan ${ }^{\mathbf{e}}$, Centre for Health and Social Care Research, Sheffield Hallam University, Collegiate Crescent, Sheffield, UK, ${ }^{b}$ Singapore Eye Research Institute, 'Department of Neurosurgery, Yong Loo Lin School of Medicine, dDepartment of Ophthalmology, Yong Loo Lin School of Medicine, National University of Singapore and ${ }^{e}$ Department of Emergency Medicine, National University Hospital, Singapore, Singapore
\end{abstract}

Correspondence to Charmaine Childs, PhD, MPhil, BNurs, Centre for Health and Social Care Research, Sheffield Hallam University, 32 Collegiate Crescent, Sheffield S10 2BP, UK

Tel: + 44 (0) 114225 2282; fax: + 44 (0) 114225 4377; e-mail: c.childs@shu.ac.uk

Received 29 January 2014 Accepted 11 April 2014

Mild traumatic brain injury (mTBI) accounts for $90 \%$ of all brain trauma. As only $15 \%$ of mTBI patients will have an identifiable intracranial lesion on brain computed tomography [1], injury is often considered as 'insignificant' when compared with the devastating and highly visible sequelae that follows severe TBI. Nevertheless, the lingering postconcussive symptoms (PCS) reported by patients after TBI are now well recognized and particularly so in returning Operation Iraqi and Operation Enduring Freedom veterans [2]. However, how can the risk of PCS be assessed in the absence of a diagnostic test? Of relevance here, and in the context of a potential candidate clinical 'biomarker', is the relationship already

This is an open-access article distributed under the terms of the Creative Commons Attribution-NonCommercial-NoDerivatives 3.0 License, where it is permissible to download and share the work provided it is properly cited. The work cannot be changed in any way or used commercially. described between retinal microvascular signs (e.g. retinopathy, retinal venular widening, increased arteriolar and venular tortuosity) and brain damage because of cerebral ischaemia after stroke [3] and cardiovascular disease [4]. To our knowledge, no study has explored the potential utility of retinal microvascular signs using retinal photography after mTBI. Here, we describe the results of a 'first of kind' pilot clinical investigation.

After obtaining ethics institutional review board approval, patients between the ages of 21 and 70 years (Glasgow Coma Scale 13-15) presenting with two or more PCS and warranting overnight hospital observation were eligible. Uncooperative or confused patients and those with preexisting comorbidity known to incur pathological changes in cerebral vessels were excluded. Optic disc-centred and macula-centred retinal fundus images were taken from each eye using a nonmydriatic digital camera (Canon CRDGi with a 20D SLR back; Canon, Tokyo, Japan) after pupil dilatation with $1 \%$ tropicamide eye drops (Mydriacyl; Alcon Pte Ltd., Singapore) following a standard protocol. A trained grader, masked to the participants' characteristics, performed the grading on the retinal images at the Singapore Eye Research Institute (SERI) Image Reading Centre. Quantitative assessment of the retinal microvascular network and retinal vascular parameters (vessel calibre, fractal dimensions, tortuosity and bifurcation) were measured using a semiautomated computer-based program (Singapore I Vessel Assessment) [5]. All mTBI patients recruited into the study were invited to return to the emergency department (ED) for repeat retinal photography at the 6-month time-point after recruitment.

Over 5 months, 43 patients who presented to the ED with mTBI were screened for study inclusion. The majority had one or more comorbidities or had one PCS only and therefore did not fulfil the eligibility criteria. Of the eligible patients (within age range, no pre-existing comorbidity, $n=9$ ), only four adult men (Indian, Chinese, Malay race) aged 21-44 (median 24.5) years provided their consent for retinal photography on admission to the ED and at followup and the bimonthly telephone assessment. To appreciate the appearance and values for retinal vascular parameters (retinal signs) of mTBI patients, data from an existing healthy control group, and age-matched, sex-matched and race-matched to the patients, were compared. We found that arteriolar and venular tortuosity was significantly increased after mTBI (Table 1). These findings remained consistent after additional adjustment for age in an analysis of covariance model. For the remaining retinal vascular parameters investigated (calibre, fractal dimensions and branching angle, Table 1), no significant differences were observed between mTBI patients and the matched controls. None of the recruited patients had signs of retinopathy. Only one patient attended for follow-up retinal images at the 6-month time-point. Here, a slight reduction in retinal vascular calibre for both eyes (right eye $217 \mu \mathrm{m}$ at 
Table 1 Comparison of retinal vascular parameters: for patients with mTBI and age group-sex-race-matched controls

\begin{tabular}{|c|c|c|c|c|c|c|}
\hline \multirow[b]{2}{*}{ Retinal vascular parameters } & \multicolumn{2}{|c|}{ Mean (SD) } & \multirow[b]{2}{*}{$P^{\star}$} & \multicolumn{2}{|c|}{ Age-adjusted mean (SD) } & \multirow[b]{2}{*}{$P^{\dagger}$} \\
\hline & mTBI & Controls & & mTBI & Controls & \\
\hline \multicolumn{7}{|l|}{ Calibre ( $\mu \mathrm{m})$} \\
\hline Arteriolar & $130.0(10.4)$ & $138.6(9.4)$ & 0.142 & $129.9(5.0)$ & $138.6(2.9)$ & 0.153 \\
\hline Venular & $201.1(13.6)$ & $208.9(15.7)$ & 0.391 & $201.2(7.9)$ & $208.8(4.6)$ & 0.424 \\
\hline \multicolumn{7}{|l|}{ Fractal dimensions } \\
\hline Arteriolar & $1.246(0.056)$ & $1.223(0.056)$ & 0.497 & $1.248(0.028)$ & $1.222(0.016)$ & 0.436 \\
\hline Venular & $1.261(0.064)$ & $1.217(0.054)$ & 0.198 & $1.265(0.026)$ & $1.216(0.015)$ & 0.125 \\
\hline \multicolumn{7}{|l|}{ Tortuosity $\left(\times 10^{-4}\right)$} \\
\hline Arteriolar & $0.927(0.176)$ & $0.595(0.117)$ & 0.001 & $0.920(0.064)$ & $0.597(0.037)$ & 0.001 \\
\hline Venular & $1.047(0.166)$ & $0.683(0.101)$ & $<0.001$ & $1.053(0.058)$ & $0.681(0.033)$ & $<0.001$ \\
\hline \multicolumn{7}{|l|}{ Branching angle } \\
\hline Arteriolar & $81.9(8.5)$ & $86.8(12.7)$ & 0.488 & $82.1(6.2)$ & $86.8(3.6)$ & 0.521 \\
\hline Venular & $82.7(4.3)$ & $73.2(10.7)$ & 0.111 & $82.6(5.0)$ & $73.2(2.9)$ & 0.127 \\
\hline
\end{tabular}

Significant $P$ values are given in bold.

mTBI, mild traumatic brain injury.

${ }^{*}$ Comparison of mean values (Student's $t$-test).

${ }^{\dagger}$ Comparison of age-adjusted mean values (analysis of covariance).

baseline vs. $211 \mu \mathrm{m}$ at the 6-month follow-up; and left eye 225 vs. $219 \mu \mathrm{m}$ ) was noted, but other vascular parameters remained unchanged. By superimposing the baseline and 6-month retinal images, no differences in network density and vessel trajectories were noted.

\section{Discussion}

To our knowledge, this is the first preliminary account to suggest that mTBI may be associated with quantifiable changes in the retinal vessels. Here, the changes we report refer specifically to increased arterial and venous tortuosity, changes that may persist for a period of 6 months from the time of injury. Previous work at SERI has shown that retinal signs (e.g. retinopathy) and the calibre of small vessels (arterioles and venules) reflect changes in cerebral small vessels [3-5]. This cerebral vascular pathology is not detectable using current routine radiographic, computed tomographic imaging or gross clinical examination. However, by performing retinal photography, subtle retinal vascular network changes are found in ischaemic stroke, dementia, cognitive dysfunction and age-related cerebrovascular disease. This work links retinal vessel pathology with cerebral vessel pathology. In this context there is a possibility for the future to explore further the links between persistent somatic, cognitive and emotional post-TBI symptoms, with abnormal retinal vessel pathology, a diagnostic capability that could be used in the future in the ED by a suitably trained technician and with the potential to screen for those patients who may be at risk of prolonged morbidity after mTBI.

We may, on the basis of the early stage report, posit a hypothesis for retinal imaging as a surrogate for cerebral vessel pathology worthy of further investigation in patients with mTBI sequelae. No untoward effects occurred during the imaging procedure; the method was safe in the setting of the ED for mTBI patients who had the capacity to provide informed consent.

\section{Acknowledgements}

The authors acknowledge the nurses and doctors of the Emergency Department of National University Hospital, Singapore, and the assistance of Tint Phyo Wai, Bernard $\mathrm{Ng}$, for assistance with data collection. The authors also thank Professor Wong Tien Yin for supporting this preliminary investigation.

\section{Conflicts of interest}

There are no conflicts of interest.

\section{References}

1 Jagoda AS. Mild traumatic brain injury: key decisions in acute management. Psychiatr Clin North Am 2010; 33:797-806.

2 Rosenfeld JV, Ford NL. Bomb blast, mild traumatic brain injury and psychiatric morbidity: A review. Injury 2010; 41:437-443.

3 Ong YT, De Silva DA, Cheung CY, Chang HM, Chen CP, Wong MC, et al. Microvascular structure and network in the retina of patients with ischaemic stroke. Stroke 2013; 44:2121-2127.

4 Wong TY, Klein R, Klein BE, Tielsch JM, Hubbard L, Nieto FJ. Retinal microvascular abnormalities and their relationship with hypertension, cardiovascular disease, and mortality. Surv Ophthalmol 2001; 46:59-80.

5 Cheung CY, Tay WT, Mitchell P, Wang JJ, Hsu W, Lee ML, et al. Quantitative and qualitative retinal microvascular characteristics and blood pressure. J Hypertens 2011; 29:1380-1391.

\section{Dexmedetomidine in addition to benzodiazepine-based sedation in patients with alcohol withdrawal delirium}

Scott Lucyk, Robert S. Hoffman and Lewis S. Nelson, Department of Emergency Medicine, Division of Medical Toxicology, New York University School of Medicine, New York, New York, USA

Correspondence to Scott Lucyk, MD, Department of Emergency Medicine, Division of Medical Toxicology, New York University School of Medicine, New York, NY, USA

Received 23 March 2014 Accepted 23 June 2014

We read with great interest the recent article [1] assessing the utility of adding dexmedetomidine to benzodiazepines in delirium tremens (DTs). DTs is a potentially life-threatening condition that requires prompt and 